\section{Digitale Kunst}

\section{Thomas Ludwig ${ }^{1}$ Michael Resch ${ }^{2}$}

Angenommen: 12. Januar 2021

(c) Springer-Verlag GmbH Deutschland, ein Teil von Springer Nature 2021

Was hat Kunst mit Informatik zu tun? Diese Frage stand im Zentrum unserer Diskussion als wir begannen dieses Sonderheft zu entwickeln. Zu klären war zunächst, wie breit das Thema angesetzt werden kann oder muss. Bei unseren Recherchen stießen wir auf eine Reihe von Entdeckungen, die wir so nicht erwartet hatten.

Betrachtet man das Thema historisch, so tastet man sich zurïck in die Geschichte des Computers und darüber hinaus die Geschichte der Maschine im Dienst der Kunst. Betrachtet man es unter dem Aspekt der Kunstgattung, so findet man in allen Bereichen der Kunst - bildende Kunst, darstellende Kunst, Musik und Literatur - die Spuren des Computers. Betrachtet man es unter dem Aspekt der Wissenschaftsdisziplin Informatik, so reicht das Spektrum von der Visualisierung über die Digital Humanities bis zu Fragen der Datenarchivierung und der Computerspiele. Zuletzt zeigt die Betrachtung des Computers als Werkzeug der Kunst die Vielfalt an Einsatzmöglichkeiten der Maschine in der Kunst als neues, der Zeit adäquates Mittel des Ausdrucks.

Wir haben uns in diesem Heft daher dazu entschlossen, ein möglichst breites Spektrum von Sichtweisen zu präsentieren und einer Reihe von herausragenden Akteurinnen und Akteuren die Möglichkeit zu geben, ihre jeweilige Sicht auf das Digitale in der Kunst zu geben. Wie in der Erforschung des Grundwassers wollten wir durch einzelne Bohrungen nicht eine vollständige Gesamtschau des Themas ermöglichen, sondern punktuell aufzeigen, was sich im Bereich des Digitalen in der Kunst ereignet und wo zukünftige weitere Bohrungen und Explorationen einen sowohl wissenschaftli-

Thomas Ludwig

ludwig@dkrz.de

Michael Resch

resch@hlrs.de

1 Deutsches Klimarechenzentrum (DKRZ), Hamburg, Deutschland

2 Höchstleistungsrechenzentrum Stuttgart (HLRS), Stuttgart, Deutschland chen als auch künstlerischen Ertrag möglich machen könnten. Dabei lag es in der Natur der Sache, dass wir nicht alle möglichen Aspekte des Themas abdecken konnten, sondern eine Vorauswahl vornehmen mussten, die uns vielversprechend schien In diesem Sinn soll dieses Heft kein Almanach des Digitalen in der Kunst sein, sondern ein Anstoß, das Thema weiter zu verfolgen.

Zwei weitere Aspekte waren in der Arbeit zur Vorbereitung für dieses Heft für uns als Informatiker darüber hinaus überraschend. Einerseits die erstaunlich geringe Sichtbarkeit digitaler Kunst in Museen und Archiven sowie in den künstlerischen Diskussionen. Obwohl digitale Kunst seit mindestens 70 Jahren existiert und spannende Ergebnisse zeigt, und obwohl der Computer längst allgegenwärtig in unserem Alltagsleben ist, scheint digitale Kunst noch immer eine Nische zu sein. Hier stellt sich die Frage, inwieweit digitale Kunst ähnlich betrachtet wird wie die Fotografie, die auch heute noch in großen Museen als Kunstform eher wenig vertreten ist. Die Überlegung drängt sich auf, ob der technische Apparat als Werkzeug des Künstlers von der Kunstwelt nach wie vor nicht akzeptiert ist.

Andererseits ist es erstaunlich, wie wenig, 85 Jahre nachdem Walter Benjamin sich mit dem Kunstwerk im Zeitalter seiner technischen Reproduzierbarkeit beschäftigt hat, das Thema eben dieser Reproduzierbarkeit in die Diskussion über das Digitale in der Kunst eingegangen ist. Im Vordergrund der Diskussion steht weniger die digital mögliche Reproduzierbarkeit digitaler Artefakte als vielmehr der Versuch, auch digitale Werke mit Hilfe digitaler Methoden aus der digitalen - beliebig reproduzierbaren - Welt herauszuheben um ihnen den nicht-digitalen Charakter eines Originals zu geben.

Nach diesen Betrachtungen und nach vielen Diskussionen mit unterschiedlichen Akteuren in der digitalen Welt der Kunst - oder der künstlerischen Welt des Digitalen entstand das hier vorliegende Sonderheft.

Unsere Reise nimmt ihren Ausgang im Zentrum und Ursprung der Informatik. Frieder Nake, der schon 1963 mit ersten künstlerischen Versuchen am Computer begann, findet im Algorithmus den Kern der Digitalen Kunst. Früh 
bereits entsteht hier digitale Poesie. Sandra Richter, Leiterin des deutschen Literaturarchivs, die das Digitale in der Literatur zu einem ihrer neuen Thema am DLA macht, und Toni Bernhart vom Institut für Literaturwissenschaften der Universität Stuttgart führen uns in dieses Gebiet ein. Peter Weibel, der als Pionier der Medienkunst gilt, schildert die weitere Entwicklung der digitalen Kunst hin zu einer Einbeziehung des Benutzers. Eine Ausprägung davon, die uns allen bekannt ist, findet sich in den Computerspielen. Sebastian Möring, der sich intensiv mit Computerspiele und digitaler Kultur beschäftigt, ergründet die Tiefen der Computerspielkunst. Solcherart Kunst stellt hohe und neuartige Anforderungen an ihre Archivierung. Kerstin Mürer und Eléonore Bernard vom Kunsthaus Zürich erklären uns, wie das Kunsthaus Zürich diesen Herausforderungen begegnet. Hier kommen wir zur Informatik zurück. Armin Weißer, Direktor Software bei der iTernity $\mathrm{GmbH}$, beschreibt die Konzepte von Speichersystemen, die eben solche Kunstwerke zu archivieren gestatten. Wir verlassen das Digitale mit einem Beitrag von Birgit Schneider, Professorin für Medienökologie an der Universität Potsdam, die darstellt, wie Daten wieder zu analoger Kunst transformiert werden. Damit schließt sich der Kreis und kehrt zurück zum Ursprung der Frage des Digitalen in der Kunst.

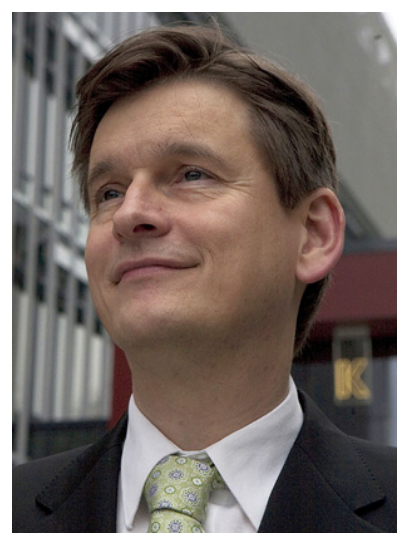

Thomas Ludwig

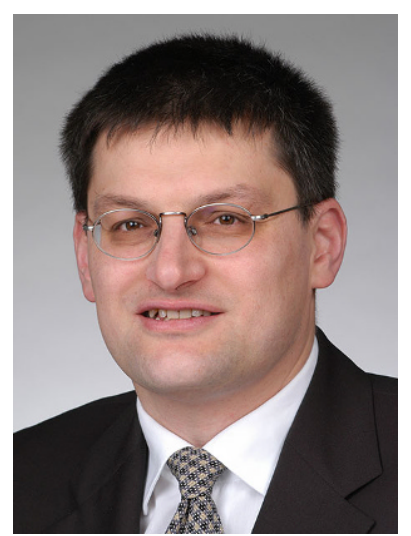

Michael Resch 